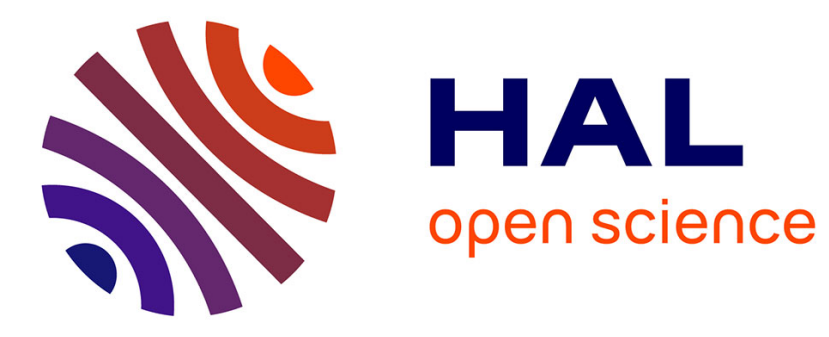

\title{
STRUCTURE DE BANDES DES ALLIAGES GaxIn1-xP
}

\author{
A. Laugier, C. Alibert, Jacques Chevallier
}

\section{To cite this version:}

A. Laugier, C. Alibert, Jacques Chevallier. STRUCTURE DE BANDES DES ALLIAGES GaxIn1xP. Journal de Physique Colloques, 1974, 35 (C3), pp.C3-77-C3-82. 10.1051/jphyscol:1974312 . jpa00215560

\section{HAL Id: jpa-00215560 https://hal.science/jpa-00215560}

Submitted on 1 Jan 1974

HAL is a multi-disciplinary open access archive for the deposit and dissemination of scientific research documents, whether they are published or not. The documents may come from teaching and research institutions in France or abroad, or from public or private research centers.
L'archive ouverte pluridisciplinaire HAL, est destinée au dépôt et à la diffusion de documents scientifiques de niveau recherche, publiés ou non, émanant des établissements d'enseignement et de recherche français ou étrangers, des laboratoires publics ou privés. 


\title{
STRUCTURE DE BANDES DES ALLIAGES Ga $\mathbf{I n}_{1-x} P$
}

\author{
A. LAUGIER \\ Laboratoire de Physique de la Matière, 20, avenue A.-Einstein, 69621 Villeurbanne, France \\ C. ALIBERT \\ Laboratoire de Physique des Solides, place E.-Bataillon, 34060 Montpellier, France

\section{J. CHEVALLIER} \\ Laboratoire de Physique des Solides 1, place A.-Briand, CNRS, 92190 Bellevue, France
}

\begin{abstract}
Résumé. - Les alliages $\mathrm{Ga}_{x} \operatorname{In}_{1-x} \mathrm{P}$ sont étudiés à $300 \mathrm{~K}$ par électroréflexion. La variation parabolique de $E_{0}$ et $E_{\mathrm{l}}$ avec $x$ est précisée, les paramètres de courbure étant respectivement $0,49 \mathrm{eV}$ et $0,86 \mathrm{eV}$. On étudie la variation de $E_{0}$ en fonction de la température entre $8 \mathrm{~K}$ et $300 \mathrm{~K}$ par piézoréflexión. On trouve une variation linéaire entre 100 et $300 \mathrm{~K}$. Le coefficient de température est $(-4,4 \pm 0,4) \mathrm{eV} / \mathrm{K}$. Aux incertitudes près, il est le même quel que soit $x$. A basse température, le paramètre de courbure de $E_{0}(x)$ vaut $0,42 \mathrm{eV}$. La bande interdite indirecte $X_{6}^{\mathrm{v}}-\Gamma_{8}^{\mathrm{v}}$ est obtenue dans la même gamme de température par électrotransmission. Les paramètres du point de croisement des minima $\Gamma$ - $X$ de la bande de conduction sont pour $T$ inférieur à $10 \mathrm{~K}, x_{\mathrm{c}}=0,69 \pm 0,015$, $E_{\mathrm{c}}=2,33 \pm 0,01 \mathrm{eV}$. A $T=300 \mathrm{~K}$ les valeurs $x_{\mathrm{c}}=0,63 \pm 0,015, E_{\mathrm{c}}=2,14 \pm 0,01 \mathrm{eV}$ sont confirmées. L'interprétation des spectres de luminescence dans le cas des dopages $\mathrm{N}$ et $\mathrm{S}$ est discutée. En particulier l'accroissement résonnant de la transition due à $\mathrm{N}$ se produit pour $x=0,65$ et non 0,71 .
\end{abstract}

\begin{abstract}
The electroreflectance spectra of $\mathrm{Ga}_{x} \mathrm{In}_{1-x} \mathrm{P}$ alloys were measured at room temperature. The variation of $E_{0}, E_{1}$ energies with concentration is parabolic. The bowing parameters are respectively $0.49 \mathrm{eV}$ and $0.86 \mathrm{eV}$. The temperature dependence of $E_{0}$ is studied by piezoreflectance from 8 to $300 \mathrm{~K}$. It is linear from $100 \mathrm{~K}$ to $300 \mathrm{~K}$ with a coefficient $\beta=(-4.4 \pm 0.4) 10^{-4} \mathrm{eV} / \mathrm{K}$ for all compositions. At low temperature the bowing parameter for $E_{0}(x)$ is $0.42 \mathrm{eV}$. In the same temperature range the $X_{6}^{\mathrm{c}}-\Gamma_{8}^{\mathrm{v}}$ indirect gap is measured by electrotransmission and the composition and the energy of the $T$ - $X$ cross-over obtained with accuracy : for $T<10 \mathrm{~K}, x_{\mathrm{c}}=0.69 \pm 0.015, E_{\mathrm{c}}=2.33 \pm 0.01 \mathrm{eV}$. At room temperature the values $x_{\mathrm{c}}=0.63 \pm 0.015, E_{\mathrm{c}}=2.14 \pm 0.01 \mathrm{eV}$ are confirmed. The interpretations of luminescence spectra of $\mathbf{S}$ and $\mathbf{N}$ doped materials are discussed. The resonant $\mathbf{N}$-trap state can exist above the fundamental conduction band edge for $x<0.65$ and not for $x<0.71$ as recently published.
\end{abstract}

1. Introduction. - Le phosphure de gallium étant un semiconducteur à grande bande interdite indirecte $\left(\Gamma_{8}^{\mathrm{v}}-\mathrm{X}_{6}^{\mathrm{c}}=2,25 \mathrm{eV}\right.$ à $\left.300 \mathrm{~K}\right)$, ses alliages avec GaAs et InP peuvent permettre d'obtenir des bandes interdites directes jusqu'à $2,06 \mathrm{eV}$ pour $\mathrm{Ga}_{x} \mathrm{As}_{1-x} \mathrm{P}$ [1] et $2,14 \mathrm{eV}$ pour $\mathrm{Ga}_{x} \mathrm{In}_{1-x} \mathrm{P}[2]$. Ce dernier système se révèle donc le plus intéressant potentiellement pour la réalisation de dispositifs électroluminescents devant fonctionner dans la zone de plus haute sensibilité de l'œil.

La structure de bande de $\mathrm{GaP}$ et InP a été calculée théoriquement [3], [4], [5] et de très nombreux paramètres ont été obtenus expérimentalement. Les ternaires $\mathrm{Ga}_{x} \mathrm{In}_{1-x} \mathrm{P}$ ne sont pas aussi bien connus. Un grand nombre d'expériences a fourni des données sur la structure de bande avec une dispersion notable due sans doute à la sensibilité médiocre de certaines techniques, à la difficulté d'interprétation des spectres et à la qualité cristalline [6]. Ceci a conduit à des désaccords parfois considérables sur la composition $x_{\mathrm{c}}$ $\mathrm{du}$ point de croisement pour lequel l'énergie des minima $\Gamma$ et $X$ de la bande de conduction devient égale.

Il est donc apparu nécessaire de déterminer avec précision l'évolution des bandes d'énergie avec la composition et la température. Pour cela nous avons choisi les techniques de modulation optiques puisqu'il est reconnu que ces méthodes sont actuellement l'outil le plus précis pour déterminer les énergies des transitions électroniques interbandes aux points critiques [7]. Les fines structures optiques obtenues sont conservées ordinairement pour les solutions solides binaires ou pseudo-binaires. A $300 \mathrm{~K}$ l'électroréflexion (ER) a été appliquée à la plupart des alliages III-V en utilisant 
la méthode de l'électrolyte, et des spectres de thermoréflexion ont été obtenus sur GaInP [8]. A basse température, la piézoréflexion (PR) et la modulation de longueur d'onde sont plus commodes. Récemment des résultats ont été obtenus sur GalnSb [10] par la première technique et sur GaAsP par la seconde [9].

Dans cet article, nous précisons la structure de bande des alliages $\mathrm{Ga}_{x} \mathrm{In}_{1-x} \mathrm{P}$ entre 8 et $300 \mathrm{~K}$ en utilisant des matériaux de haute pureté et de bonne homogénéité. Dans la section 2, les méthodes de croissance à partir de solutions, de préparation de surface et de mesures, sont décrites. Les principaux résultats obtenus section 3 et discutés section 4 sont :

i) l'étude très précise, à $300 \mathrm{~K}$, par électroréflexion de la bande interdite directe $\Gamma_{6}^{\mathrm{c}}-\Gamma_{8}^{\mathrm{v}}$, suivie dans tout le domaine de composition. Une loi parabolique $a+b x+c x(1-x)$ rend bien compte des résultats. Afin d'établir avec précision la valeur du paramètre de courbure $C$, une étude détaillée a été effectuée sur des échantillons de composition voisine de 0,5 . On trouve $C=0,49 \mathrm{eV}$;

ii) l'étude en fonction de la température de la bande interdite directe entre 8 et $300 \mathrm{~K}$ par piézoréflexion. La variation est linéaire au-dessus de $100 \mathrm{~K}$ et le coefficient de température $-4,4 \pm 0,4 \times 10^{-4} \mathrm{eV} / \mathrm{K}$ ne varie pas sensiblement avec $x$. Pour $T$ inférieur à $10 \mathrm{~K}, C=0,42 \mathrm{eV}$;

iii) l'étude de la bande interdite indirecte $X_{6}^{c}-\Gamma_{8}^{v}$ par électrotransmission dans la même gamme de température, afin de déterminer les paramètres du point de croisement, $x_{\mathrm{c}}$ et $E_{\mathrm{c}}$. Pour $T$ inférieur à $10 \mathrm{~K}$ on trouve $x_{\mathrm{c}}=0,69 \pm 0,015, E_{\mathrm{c}}=2,33 \pm 0,01 \mathrm{eV}$. Pour $T=300 \mathrm{~K}$ on confirme les valeurs

$$
x_{\mathrm{c}}=0,63 \pm 0,015, \quad E_{\mathrm{c}}=2,14 \pm 0,01
$$

précédemment obtenues [2].

On discute et on critique, section 5, l'interprétation de résultats de luminescence dans le cas des dopages $\mathbf{S}$ et $N$. En particulier, l'accroissement résonnant de la transition due à l'azote se produit à la composition $x=0,65$ et non 0,71 comme récemment publié.

2. Techniques expérimentales. - 2.1 PrÉPARATION DES ÉCHANTILLONS. - Les cristaux sont obtenus par une méthode de croissance en solution avec alimentation continue en phosphore et en gallium [11], ce qui permet d'éviter les problèmes liés à la pression de vapeur élevée du phosphore et au grand coefficient de partage du gallium. On obtient des matériaux très homogènes dans toute la gamme de composition. Typiquement on peut obtenir une variation de composition $\Delta x$, déterminée à la microsonde électronique, inférieure à 0,08 longitudinalement sur une distance de $10 \mathrm{~mm}$ et inférieure à 0,02 radialemeńt pour un diamètre de $10 \mathrm{~mm}$. Les cristaux non dopés intentionnellement ont une concentration de porteurs, mesurée par effet Hall, de l'ordre de $5 \times 10^{16}$ porteurs par centimètre cube. Ils sont de type $n$ pour $0<x<0,6$, p pour $0,6<x<1$. Ils sont légèrement compensés. Les échantillons utilisés pour cette étude sont polycristallins. Après polissage mécanique des cristaux, un léger décapage chimique est effectué, juste avant les mesures, dans un mélange d'acides chlorhydrique et nitrique $(1: 4)$ pour $x>0,4$ et un mélange de brome et de méthanol pour $x<0,4$, puis on effectue un rinçage à l'eau distillée. Bien que des grains de l'ordre du $\mathrm{mm}^{2}$ apparaissent, la surface obtenue est très brillante.

2.2 TeChNique de Modulation. - L'appareillage utilisé pour l'électroréflexion par la méthode de l'électrolyte a déjà été décrit [2]-[12]. Pour les méthodes. de piézomodulation, l'optique et le systèmede détection sont identiques au précédent. La modulation est obtenue à l'aide' de céramiques piézo-électriques sur lesquelles sont collés les échantillons. Ces céramiques sont excitées par une tension sinusoïdale de quelques centaines de volts à $600 \mathrm{~Hz}$. Pour éviter que les spectres de piézoréflexion ne soient perturbés par les réflexions multiples dans l'échantillon, nous avons choisi l'épaisseur $d$ de façon que $\alpha d>1(\alpha$ : coefficient d'absorption). L'échantillon est placé dans l'atmosphère d'un cryostat optique à circulation d'hélium gazeux. La température, mesurée par une résịstance au carbone ou une résistance au platine qui sont situées au voisinage immédiat de l'échantillon, peut être stabilisée au degré près.

3. Résultats. - 3.1 ANALYSE DES SPECTRES EXPÉRIMENTAUX. - 3.1.1 Electroréflexion. - La théorie de l'électroréflexion est actuellement bien comprise pour les champs électriques faibles pour lesquels les spectres se simplifient considérablement [13]. Ces spectres sont étroits et leur largeur en énergie n'est limitée que par le paramètre d'élargissement $\Gamma$ dû à la durée de vie de la paire électron-trou mise en jeu. Seuls ces spectres, très localisés en énergie, permettent de séparer les points critiques très voisins. L'énergie de la transition est obtenue, sans connaissance des constantes optiques et sans analyse de Kramers-Kronig, à l'aide de la méthode des trois points de Aspnes et Rowe [14]. La précision de cette méthode a été confirmée par des mesures sur le germanium [12], le phosphure d'indium et le phosphure de gallium [2].

3.1.2 Piézoréflexion. - Les spectres sont moins bien résolus. Leur interprétation théorique est délicate car elle nécessiterait la connaissance des constantes optiques. Nous les avons interprétés à $300 \mathrm{~K}$ à l'aide des mesures d'électroréflexion faites simultanément ou successivement sur le même échantillon et, à basse température, par continuité.

3.2. STRUCTURE DES BANDES D'ÉNERGIE A $300 \%$. Les principaux résultats, basés sur les mesures d'électroréflexion ont déjà été publiés [2]. L'ensemble des transitions étudiées est représenté sur la figure 1 


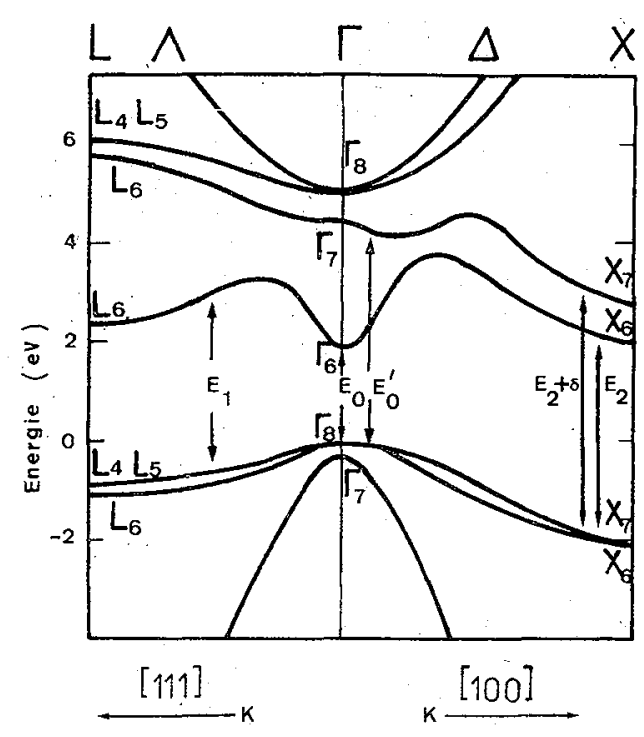

FIG, 1.- Structure des bandes d'énergie de l'alliage $\mathrm{Ga}_{x} \operatorname{In}_{1-x} \mathrm{P}$ pour $x=0,5$. Lorigine des énergies est prise au sommet de la bande de valence.

qui donne lá structure des bandes d'énergie de l'alliage $\mathrm{Ga}_{x} \operatorname{In}_{1-x} \mathrm{P}$ pour $x=0,50$.

a) La variation du gap direct $E_{0}\left(\Gamma_{6}^{\mathrm{c}}-\Gamma_{8}^{\mathrm{v}}\right)$ est bien décrite par une loi parabolique :

$$
E_{0}=1,345+1,435 x+0,50(x-1) x \quad(\mathrm{eV}) \text {. }
$$

L'éclatement spin-orbite $\Delta_{0}$ varie de façon approximativement linéaire.

$$
\begin{aligned}
& \text { b) Pour } E_{1}\left[A_{6}^{\mathrm{c}}-\left(\Lambda_{4}^{\mathrm{v}}, \mathcal{A}_{5}^{\mathrm{v}}\right)\right] \text { on a } \\
& E_{1}=3,175+0,581 x+0,860 x(x-1) \quad(\mathrm{eV}) .
\end{aligned}
$$

L'éclatement spin-orbite $\Delta_{1}$ ne varie pas de façon linéaire avec $x$. L'écart à la linéarité peut être expliqué par un glissement du point critique $A$ dans la direction $L$ de la zone de Brillouin. Récemment, Van Vechten et al. [15] ont effectué une analyse heuristique basée sur l'effet du désordre microscopique. Ils calculent un écart à la linéarité de $92 \mathrm{meV}$ ce qui est en accord avec notre résultat: $80 \pm 20 \mathrm{meV}$.

c) Les pics observés sur l'InP à $4,7 \mathrm{eV}, 5 \mathrm{eV}$ et $6 \mathrm{eV}$ et sur le GaP à 4,8 eV, 5,3 eV et 5,7 eV (avec une précision de $\pm 0,04 \mathrm{eV})$, correspondent probablement aux transitions $E_{0}^{\prime}\left(\Gamma_{7}^{\mathrm{c}}-\Gamma_{8}^{\mathrm{v}}\right), E_{2}\left(X_{1}^{\mathrm{c}}-X_{5}^{\mathrm{v}}\right)$ et

$$
E_{2}+\delta\left(X_{3}^{\mathrm{c}}-X_{5}^{\mathrm{v}}\right) \text {. }
$$

Cependant, les transitions qui peuvent donner lieu à ces structures peuvent inclure aussi $\Delta_{5}-\Delta_{1}$ et $\Sigma_{2}-\Sigma_{1}$ (dans la représentation du simple groupe) [16]-[5]. Compte teñu des incertitudes. expérimentales, on peut estimer que la variation avec $x$ de ces niveaux est linéaire.

Afin d'établir plus fermement les résultats précédents et d'écarter au maximum les possibilités d'erreurs sur la détermination de la composition $x$ et sur les effets d'hétérogénéité, nous avons procédé de la manière suivante. D'une part, des mesures de composition ont été effectuées, par microsonde électronique, sur un même cristal par l'appareil déjà utilisé (CNRS, Bellevue) et par un laboratoire américain indépendant [17]. Elles ont révélé que nos mesures étaient décalées systématiquement de $\Delta x=0,01$ vers les fortes compositions. D'autre part, afin de minimiser les effets d'hétérogénéité, nous avons fait des mesures d'électroréflexion, de pićzoréflexion, d'électro-absorption et de luminescence sur des surfaces de l'ordre de $1 \mathrm{~mm}^{2}$, la composition étant donnée avec une incertitude inférieure ou égale à 0,005 .

La figure 2 montre le déplacement des spectres d'électroréflexion relatifs à $E_{0}$ dû aux variations de composition d'un cristal de composition moyenne $x=0,52$. La surface du spot était inférieure à $0,5 \mathrm{~mm}^{2}$. La forme des spectres n'est pas modifiée d'un point à un autre. Ce déplacement est en bon accord avec les variations de composition mesurées à la microsonde électronique. Il est intéressant de souligner que les spectres d'électroréflexion permettent de mettre en évidence facilement, des fluctuations de composition inférieures à 0,005 . Pour $x=0,527$ la valeur de la bande interdite est de $1,980 \pm 0,004 \mathrm{eV}$, le paramètre de courbure de l'éq. (1) devient $c=0,49 \pm 0,01$. Les résultats précédents (2) sont donc confirmés.

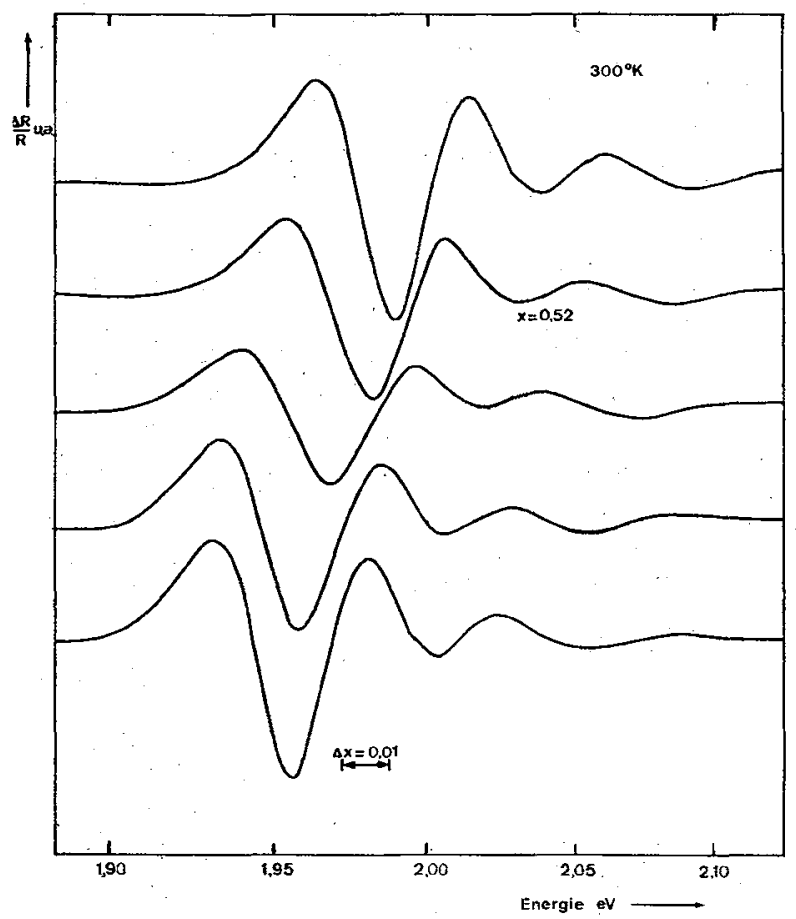

Frg. 2. - Sprectre d'ER à $300 \mathrm{~K}$ au voisinage de la bande interdite directe d'un échantillon de $\mathrm{Ga}_{x} \operatorname{In}_{1-x} \mathrm{P}$ ayant une composition moyenne $x$ égale à 0,52 . Ces spectres sont obtenus en focalisant un spot lumineux de $0,5 \mathrm{~mm}^{2}$ sur différents points de la surface de l'échantillon. On remarque qu'une différence de composition inférieure à 0,005 peut être décelée par ER. Le glissement des spectres correspondant à une variation $\Delta x=0,01$ de la composition est aussi inđiqué. 
3.3 ETUde A BASSE TEMPÉRATURE. - La figure 3 montre l'évolution en fonction de la température des spectres de piézoréflexion pour une composition $x=0,51$. La variation de la bande interdite pour $x=0,51$ et $x=0,28$ est donnée sur la figure 4 . On note que la variation est linéaire au-dessus de $100 \mathrm{~K}$ et est représentée alors par la loi :

avec

$$
E_{0}(T)=E_{0}(0)+\beta T
$$

et

$$
\beta=4,3 \times 10^{-4} \mathrm{eV} / \mathrm{K}(x=0,28)
$$$$
\beta=-4,6 \times 10^{-4} \mathrm{eV} / \mathrm{K}(x=0,51) \text {. }
$$

L'incertitude sur $\beta$ étant de $\pm 0,4 \times 10^{-4} \mathrm{eV} / \mathrm{K}$.

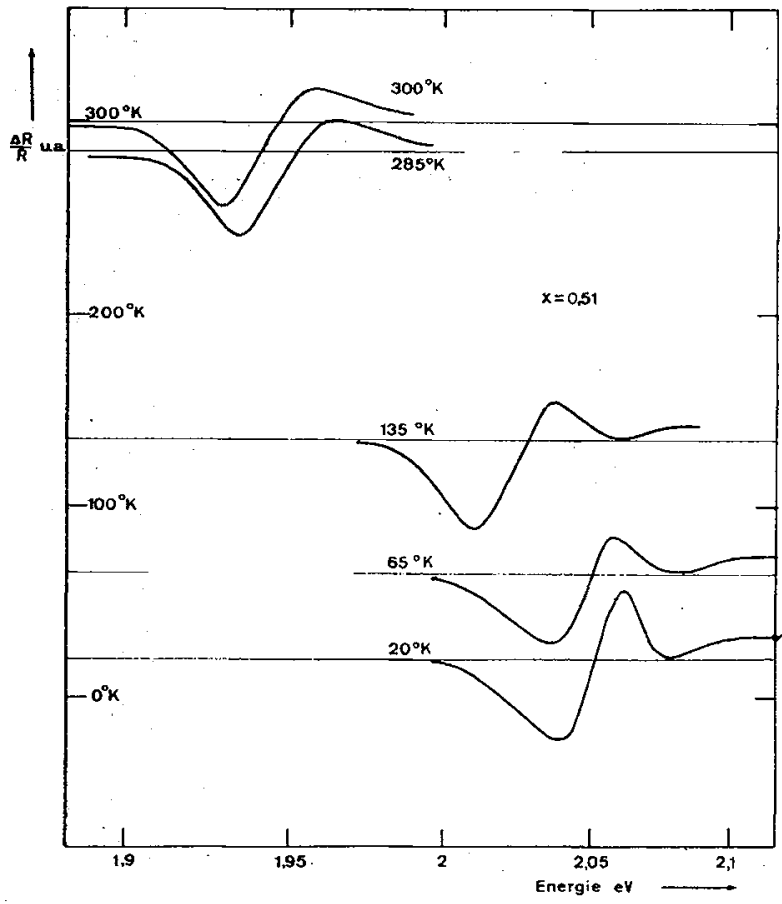

Fig. 3. - Evolution en fonction de la température des spectres de $P R$ au voisinage de la bande interdite directe d'un échantillon de $\mathrm{Ga}_{x} \operatorname{In}_{1-x} \mathrm{P}(x=0,51)$. Le décalage en ordonnée est proportionnel à la température.

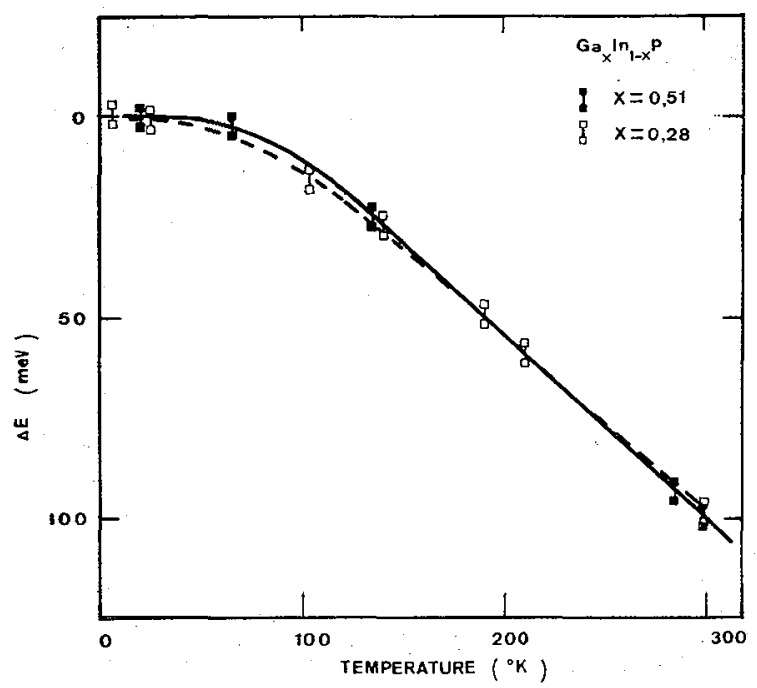

Frg. 4. - Variation relative de l'énergie de la bande interdite directe en fonction de la température pour $x=0,51$ et $x=0,28$.
4. Discussion. - 4.1 Paramètre de Courbure. La précision sur la valeur du paramètre de courbure de la bande interdite directe donnée en 3 n'est limitée que par l'incertitude liée aux mesures de composition. Vers $2 \mathrm{eV}$ la structure due au couplage spin-orbite n'influe pas sensiblement sur la valeur théorique de la bande interdite. En effet, les spectres d'ER s'étendent en énergie sur une largeur de 3 fois le paramètre d'élargissement $\Gamma(\Gamma=30 \mathrm{meV}$ à $300 \mathrm{~K})$ alors que $\Delta_{0}$ est de l'ordre de $100 \mathrm{meV}$ et que l'amplitude du spectre due à $\Delta_{0}$ est 5 fois plus faible. Les structures $E_{0}$ et $E_{0}+\Delta_{0}$ n'interfèrent, très peu, que sur le troisième pic dû à $E_{0}$, qui, de toute façon n'est pas utilisé dans la détermination de la bande interdite par la méthode des trois points.

Van Vechten et Bergstresser [18] ont estimé, à l'aide d'un modèle intuitif les valeurs de $C$ comme déjà indiqué [2], les valeurs expérimentales ne concordent pas bien avec cette estimation. Séparant $C$ en deux parties : $C_{\mathrm{i}}$ trouvée dans l'approximation du cristal virtuel,' $C_{\mathrm{e}}$ dû aux effets extrinsèquès (désordre, apériodicité, etc...) on calcule pour $E_{0}$ :

$$
C_{\mathrm{i}}=0,37, \quad C_{\mathrm{e}}=0,31, \quad C=0,68,
$$

plus grand que la valeur expérimentale 0,49 ; pour $E_{1}$ on a :

$$
C_{\mathrm{i}}=0,27, \quad C_{\mathrm{e}}=0,31, \quad C=0,58 .
$$

plus petit que la valeur expérimentale 0,86 . (Ces valeurs sont exprimées en eV.) Dans le cas de GaAsP, Stroud [19] a amélioré le modèle sur la base d'un calcul de perturbation, suggérant que $C_{\mathrm{e}}$ peut être, en fait, différent pour différentes bandes, ce qui est indispensable pour rendre compte des résultats sur GaInP. Cependant l'écart important observé pour ce système suggère qu'une méthode de perturbation au second ordre n'est pas valable. Ces conclusions, valables à $300 \mathrm{~K}$, ne sont pas modifiées à basse température.

4.2 STRUCTURE DE BANDE A BASSE TEMPÉRATURE. Les coefficients de température déterminés par les alliages sont du même ordre de grandeur que ceux des constituants, aux incertitudes expérimentales près. En ce qui concerne les mesures de PR, les incertitudes proviennent essentiellement $\mathrm{du}$ fait que nous avons déterminé la position de la bande interdite par continuité,'alors qu'il a été montré [20] qu'un paramètre fondamental de la forme des spectres de PR est le champ électrique dont l'évolution n'est pas connue en fonction de la température.

La variation de la bande interdite avec la température provient d'une part, de la contribution de la dilatation thermique, d'autre part, et c'est.l'effet déterminant, des interactions électrons-phonons. Un récent calcul de ces effets [21] donne pour

$$
\mathrm{GaP} \beta=-4,5 \times 10^{-4} \mathrm{eV} / \mathrm{K}
$$

et pour

$$
\operatorname{InP} \beta=-4 \times 10^{-4} \mathrm{eV} / \mathrm{K}
$$

en accord avec les valeurs expérimentales. Pour les alliages, les valeurs de $\beta$ sont du même ordre de gran- 
deur de sorte que le paramètre de courbure de $\Gamma_{6}^{\mathrm{c}}-\Gamma_{8}^{\mathrm{v}}$ varie peu entre 8 et $300 \mathrm{~K}$. Connaissant les valeurs des bandes interdites directes de $\mathrm{GaP}$ et InP, en dessous de $10 \mathrm{~K}: 2,878 \mathrm{eV}$ [23], 1,423 eV [22], on trouve $C=0,42 \mathrm{eV}$.

4.3 PoINT DE CROISEMENT. - La variation de $X_{6}^{\mathrm{c}}-\Gamma_{8}^{\mathrm{v}}$ avec la composition est assez fortement modifiée par la température [2] de sorte que la composition du point de croisement varie beaucoup avec la température. Comme les mesures d'ER et de PR ne peuvent pas donner, compte tenu de l'épaisseur des échantillons, des renseignements sur la bande interdite indirecte $E_{\mathrm{gx}}$, nous avons effectué des mesures d'électrotransmission sur un échantillon de composition $x=0,77$, à 77 et $300 \mathrm{~K}$ en prenant $\alpha d<1$. Les spectres obtenus sont donnés sur la figure 5 qui montre la. variation importante de $E_{g x}$ avec $x$ à $300 \mathrm{~K}$. On mesure

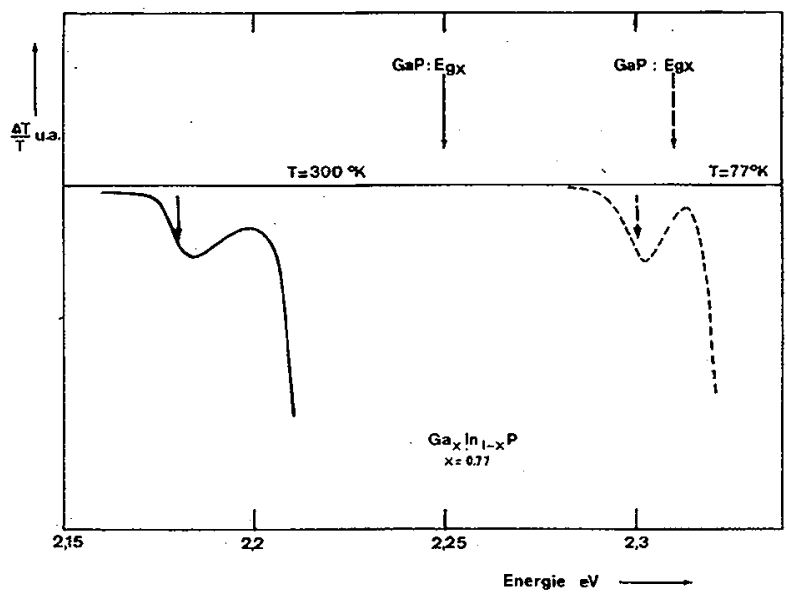

FIG. 5. - Spectres d'EA au voisinage de la bande interdite indirecte à $T=300 \mathrm{~K}$ et $T=77 \mathrm{~K}$ pour $x=0,77$. La bande interdite indirecte du GaP à $300 \mathrm{~K}$ et $77 \mathrm{~K}$ est aussi indiquée.

une pente $\mathrm{d} E_{\mathrm{g}_{x}} / \mathrm{d} x$ de $3 \mathrm{meV} /$ at $\%$, en excellent accord avec les valeurs précédemment obtenues par absorption optique [2], [24]. Par contre, à basse température, la pente est faible, on mesure $0,4 \mathrm{meV} / \mathrm{at} \%$. En supposant que cette pente est la même que celle de la transition excitonique observée sur des matériaux dopés au soufre (exciton lié au soufre neutre) on obtient la même valeur.

A $300 \mathrm{~K}$ le présent travail confirme donc les valeurs $x_{\mathrm{c}}=0,63 \pm 0,015$ et $E_{\mathrm{c}}=2,14 \pm 0,01 \mathrm{eV}$ [2]. En dessous de $10 \mathrm{~K}$, on trouve $x_{\mathfrak{c}}=0,69 \pm 0,015$ et

$$
E_{\mathrm{c}}=2,33 \pm 0,01 \mathrm{eV} \text {. }
$$

5. Luminescence. - La structure de bande étant connue à toute température et composition, nous pouvons interpréter les transitions observées en photoluminescence. La figure 6 présente le spectre obtenu à $4,2 \mathrm{~K}$ dans les conditions déjà précisées, sur une partie très localisée d'un alliage non intentionnellement dopé. On voit que l'énergie de la bande interdite est supérieure à celle du pic « haute énergie ». Des spectres tout à fait similaires sont obtenus pour $0<x<0,6$ [25].
Une étude plus approfondie de l'évolution de ce spectre avec la température [26] conduit à interpréter le pic de plus haute énergie par une transition entre un donneur et la bande de valence et l'autre pic par une transition de paires donneur-accepteur. L'énergie d'ionisation du donneur est difficile à préciser en raison du cumul des incertitudes propres aux mesures indépendantes de photoluminescence, d'ER et de PR. D'après l'évolution du spectre en fonction du niveau de dopage et d'après les propriétés électriques des alliages [26] on peut estimer que l'énergie d'ionisation du donneur est inférieure à l'écart énergétique de $40 \mathrm{méV}$ (pour $x=0,52$ ) trouvé entre la valeur de $E_{\mathrm{g}}$ et la position du pic haute énergie.

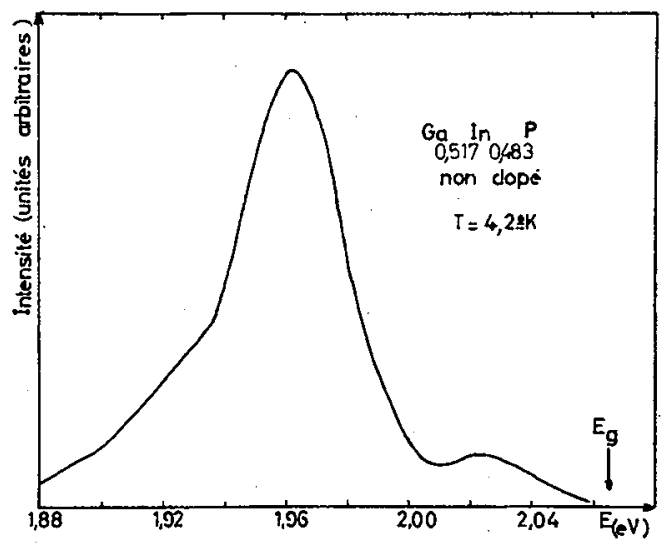

Fig. 6. - Photoluminescence d'un alliage non intentionnellement dopé à bande interdite directe. La flèche indique l'énergie de la bande interdite déduite des mesures de PR à $10 \mathrm{~K}$ environ.

Sur la figure 7 nous avons porté la variation de $E_{\mathrm{g}}(x)$ ainsi que quelques résultats de photoluminescence afin de les comparer à ceux de Macksey et al. [27] dont les matériaux sont dopés Te et $\mathrm{N}$. Pour $x<0,68$ leurs spectres sont très analogues aux nôtres. Prenant comme bande interdite directe $E_{\mathrm{g}_{\Gamma}}$ les valeurs données par Onton et al. [1] ces auteurs interprètent le pic haute énergie comme étant dû à un état résonnant đu piège azote dans la bande de conduction, le pic basse énergie

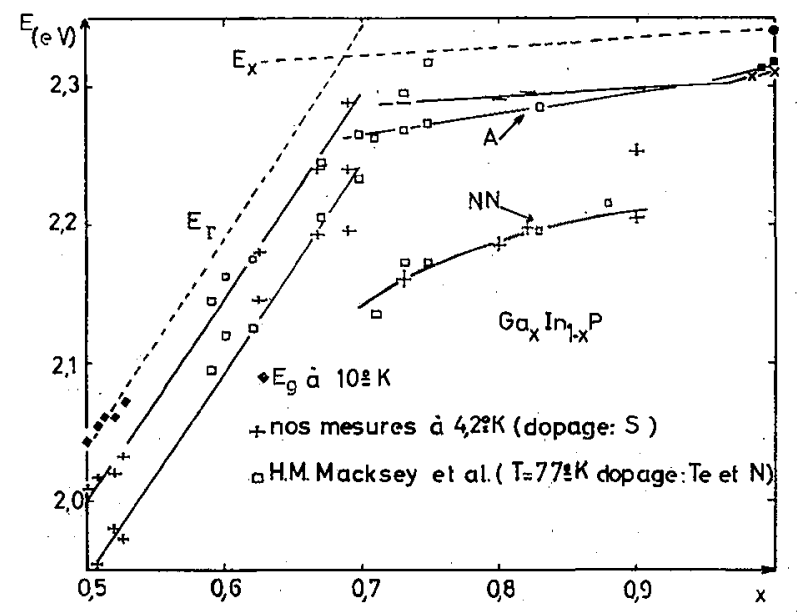

FIG: 7. - Transition de luminescence des alliages $G_{a x} \operatorname{In}_{1-x} \mathbf{P}$ et variation des bandes interdites. 
étant la transition bande à bande. A partir de nos mesures d'ER et de PR nous pouvons réinterpréter ces deux pics comme nous l'avons fait plus haut. En outre, l'accroissement résonnant de la transition $\mathrm{A}$ dû à l'azote se produit à la composition telle que $E_{\mathrm{A}} \simeq E_{\mathrm{g}_{\Gamma}}$. Compte tenu des présents résultats, cette composition $x_{0}$ est égale à 0,65 au lieu de 0,71 comme le prévoient Macksey et al. Ces effets de résonance sont donc beaucoup moins importants que ceux décrits par ces auteurs. Ils sont beaucoup moins marqués pour GaInP que pour GaAsP, ce qui est tout à fait normal puisque $\operatorname{Grad}_{x}\left(E_{\Gamma}-E_{\mathrm{X}}\right)$ est plus grand.

\section{Bibliographie}

[1] Onton, A., Lorentz, M. R. et Reuter, W., J. Appl. Phys. 42 (1971) 3420

[2] Alibert, C., Bordure, G., Laugier, A. et Chevallier, J., Phys. Rev. B 4 (1972) 1301.

[3] Pollak, F. H., Higginbotham, G. W. et Cardona, M., J. Phys. Soc. Japan Suppl. 21 (1966) 20.

[4] Cohen, M. L. et Bergstresser, T. K., Phys. Rev. 141 (1966) 789.

[5] Voir la référence [2] et sa bibliographie.

[6] Alvarez, C. V., Walter, J. P., Cohen, M. L., Stokes, J. et SHEn, Y. R., Phys. Rev. B 6 (1972) 1412.

[7] Cardona, M., Modulation Spectroscopy (Academic N. Y.), 1969.

[8] Lettington, A. H., Jones, D. et Sarginson, R. S., J. Phys. C. 4 (1971) 1534.

[9] Onton, A. et Foster, L. M., J. Appl. Phys. 43 (1972) 5083.

[10] Auvergne, D., Camassel, J., Mathieu, H. et Joullie, A., J. Phys. \& Chem. Solids 1973 (à paraître).

[11] Laugier, A. et Chevallier, J., Phys. Stat. Sol. 7 (1971) 427.

[12] Alibert, C., Thèse d'Etat, Montpellier, 1973.

[13] Aspnes, D. E., Surface Science 37 (1973) 418.

[14] Aspnes, D. E. et Rowe, J. E., Phys. Rev. Lett. 4 (1971) 188.

[15] Van Vechten, J. A., Berolo, O. et Woolley, J. C., Phys. Rev. Lett. 29 (1972) 1400.
[16] Stokowski, S. E. et Seld, D. D., Phys. Rev. B 5 (1972) 1636.

[17] Lab. IBM (Yorktown Heights, N. Y.). Nous tenons à remercier le Dr ONTON pour ces analyses.

[18] Van Vechten, J. A. et Bergstresser, T. K.; Phys. Rev. B 1 (1970) 3351 .

[19] Stroud, D., Phys. Rev. B 5 (1972) 3366.

[20] Alibert, C., à paraître.

[21] Tsay, Y. F., Gong, B., Mitra, S. S. et Vetelino, J. F., Phys. Rev. B 6 (1972) 2330.

[22] White, A. M., Dean, P. J., Taylor, L. L., Clarke, R. C., Ashen, D. J. et Mullini J. B., J. Phys. C. 5 (1972) 1727.

[23] Dean, P. J., Kaminsky, G. et Zetterstrom, R. B., J. Appl. Phys. 38 (1967) 3551.

[24] Hakki, B. W., JaYaraman, A. et Kim, C. K., J. Appl. Phys. 41 (1970) 5291.

[25] Chevallier, J. et Laugier, A., Phys. Stat. Sol. (a) 8 (1971) 437.

[26] Chevallier, J., Propriétés optiques des Semiconducteurs à grande bande interdite. Montpellier, septembre 1973.

[27] Macksey, H. M., Holonyak, Jr. N., Dupuis, R. D., Campeell, J. C. et ZACK, G. W., J. Appl. Phys. 44 (1973) 1333.

\section{DISCUSSION}

D. AUVERGNE. - En piézoréflection, vous déterminez le gap à basse température à partir de la comparaison avec les spectres d'électroréflectance à la température ambiante. Tenez-vous compte de la variation de largeur du spectre de piézoréflectance avec la température ?

A. Laugier. - Pour l'évaluation du gap, il a en effet été tenu compte des variations de la forme et de la largeur des spectres de piézoréflectance.

H. KRESSEL. - What is the carrier concentration in your samples and what are the residual dopants?

A. LAUGIER. - The carrier concentration is $5 \times 10^{16} \mathrm{~cm}^{-3}$. In undoped materials the residual impurities are : $\mathrm{C}, \mathrm{N}, \mathrm{O}, \mathrm{Si}, \mathrm{Na}, \mathrm{K}(\simeq \mathrm{ppm})$.

P. J. DEAN. - Recent measurements of the dependence of electrical transport on hydrostatic pressure by Pitt and coworkers at STL using vpe constant composition layers grown by Mabbitt and coworkers at Plessey suggest an important modification to the current view of the direct-indirect band cross-over in $\mathrm{Ga}_{x} \mathrm{In}_{1-x} \mathrm{P}$. Preliminary interpretation of their data, using known pressure coefficients for bandgaps in
III-V compounds of this type, suggests that the crossover near $x=0.73$ at $300 \mathrm{~K}$ reported by some authors occurs between the $\mathrm{X}$ and $\mathrm{L}$ indirect minima, not $\mathrm{X}$ and $\Gamma$ as suggested by some previous workers, notably the group at IBM. According to the new work, the $\mathrm{X}-\Gamma$ and $\mathrm{L}-\Gamma$ band cross-overs both lie near $x=0.63$, with the former at slightly larger $x$. These data provide a means of reconciling the mysterious inconsistencies in interpretations of previous measurements on a variety of physical properties, often on alloys of well controlled composition. These inconsistencies were peculiar to this alloy system, in which the band structure is peculiarly complicated, as compared with $\mathrm{GaAs}_{x} \mathrm{P}_{1-x}$ for example. The kink in the composition dependence of the $\mathrm{N}$ level just below $x=0.7$, shown in the last figure of the present talk is also naturally explicable in terms of an interaction between $\mathrm{N}$ states derived from the $\mathrm{X}$ and $\mathrm{L}$ minima, visible when the $\mathrm{N}$ level and $\mathrm{L}$ minima approach a cross-over. It is entirely unexpected if the $\mathrm{X}$ and $\Gamma$ minima alone cross near this composition, as verified experimentally in the composition dependence of the $\mathrm{N}$ level in $\mathrm{GaAs}_{x} \mathbf{P}_{1-x}$ where the $\mathrm{L}$ minima remain far above the $\mathrm{X}$ over the whole range of $x$. 